\title{
EXPERIENCE DEMOGRAPHIC FACTORS AND THEIR INFLUENCE ON INSTRUCTOR PERSPECTIVES OF ONLINE VERSUS FACE-TO-FACE EDUCATION AT A JESUIT INSTITUTION
}

\author{
Lynn A. Fish and Coral R. Snodgrass \\ The BRC Academy Journal of Business 9, no. 1 (2019): 75-100. \\ http://dx.doi.org/10.15239/j.brcacadjb.2019.09.01.ja05 \\ Web APPENDIX \\ http://dx.doi.org/10.15239/j.brcacadjb.2019.09.01.wa05
}

Table 1. Pearson Correlation Factors

\begin{tabular}{|c|c|c|c|c|c|c|}
\hline OL Instructors & $\begin{array}{l}\text { Level } \\
\text { Taught }\end{array}$ & $\begin{array}{l}\text { \# Years } \\
\text { Taught } \\
\text { University }\end{array}$ & $\begin{array}{l}\text { \# Years } \\
\text { Taught } \\
\text { Total }\end{array}$ & $\begin{array}{l}\text { Online } \\
\text { Experience } \\
\text { University }\end{array}$ & $\begin{array}{l}\text { Online } \\
\text { Experience } \\
\text { Other }\end{array}$ & $\begin{array}{l}\text { Self- } \\
\text { reported } \\
\text { Technology } \\
\text { Level }\end{array}$ \\
\hline Level Taught & 1 & & & & & \\
\hline $\begin{array}{l}\text { \# Years Taught } \\
\text { University }\end{array}$ & -.289 & 1 & & & & \\
\hline \# Years Taught Total & -.204 & $.751 *$ & 1 & & & \\
\hline Online Experience & .066 & -.298 & -.038 & 1 & & \\
\hline $\begin{array}{l}\text { Online Experience } \\
\text { Other }\end{array}$ & -.272 & .295 & .006 & $-.311 * *$ & 1 & \\
\hline Self-reported Tech. & .045 & -.105 & -.008 & -.154 & -.227 & 1 \\
\hline
\end{tabular}




\begin{tabular}{|c|c|c|c|c|c|c|}
\hline Level & & & & & & \\
\hline FTF Instructors & $\begin{array}{l}\text { Level } \\
\text { Taught }\end{array}$ & $\begin{array}{l}\text { \# Years } \\
\text { Taught } \\
\text { University }\end{array}$ & $\begin{array}{l}\text { \# Years } \\
\text { Taught } \\
\text { Total }\end{array}$ & $\begin{array}{l}\text { Online } \\
\text { Experience }\end{array}$ & $\begin{array}{l}\text { Online } \\
\text { Experience } \\
\text { other }\end{array}$ & $\begin{array}{l}\text { Self- } \\
\text { reported } \\
\text { Technology } \\
\text { Level }\end{array}$ \\
\hline Level Taught & 1.0000 & & & & & \\
\hline $\begin{array}{l}\text { \# Years Taught } \\
\text { University }\end{array}$ & -.120 & 1 & & & & \\
\hline \# Years Taught Total & -.120 & $.947 *$ & 1 & & & \\
\hline Online Experience & .060 & -.145 & -.152 & 1 & & \\
\hline $\begin{array}{l}\text { Online Experience } \\
\text { Other }\end{array}$ & -.144 & -.017 & -.030 & -.004 & 1 & \\
\hline $\begin{array}{l}\text { Self-reported Tech. } \\
\text { Level }\end{array}$ & .261 & -.250 & -.116 & -.179 & -.263 & 1 \\
\hline Overall & $\begin{array}{l}\text { Level } \\
\text { Taught }\end{array}$ & $\begin{array}{l}\text { \# Years } \\
\text { Taught } \\
\text { University }\end{array}$ & $\begin{array}{l}\text { \# Years } \\
\text { Taught } \\
\text { Total }\end{array}$ & $\begin{array}{l}\text { Online } \\
\text { Experience }\end{array}$ & $\begin{array}{l}\text { Online } \\
\text { Experience } \\
\text { Other }\end{array}$ & $\begin{array}{l}\text { Self- } \\
\text { reported } \\
\text { Technology } \\
\text { Level }\end{array}$ \\
\hline Level Taught & 1.0000 & & & & & \\
\hline $\begin{array}{l}\text { \# Years Taught } \\
\text { University }\end{array}$ & -.175 & 1 & & & & \\
\hline \# Years Taught Total & -.121 & $.875 *$ & 1 & & & \\
\hline Online Experience & $-.257 * *$ & -.148 & -.101 & 1 & & \\
\hline $\begin{array}{l}\text { Online Experience } \\
\text { Other }\end{array}$ & $-.281 * *$ & .133 & -.020 & .016 & 1 & \\
\hline
\end{tabular}




\begin{tabular}{|l|l|l|l|l|l|l|}
\cline { 2 - 6 } Self-reported Tech. & .028 & -.063 & .144 & $.311^{* *}$ & -.099 & 1 \\
\hline
\end{tabular}

* Significant at .01 level (2-tailed) $\quad * *$ Significant at .05 level (2-tailed) 
Table 2. OL Instructors by Level Taught

\begin{tabular}{|c|c|c|c|c|c|}
\hline Metric & $\begin{array}{l}\text { Pearson Chi- } \\
\text { Square Value }\end{array}$ & Df & $\begin{array}{l}\text { Asymptotic } \\
\text { Significance } \\
\text { (2-sided) }\end{array}$ & $\begin{array}{c}\text { Pearson's } \\
\text { R }\end{array}$ & $\begin{array}{l}\text { Spearman } \\
\text { Correlation }\end{array}$ \\
\hline Difficulty & 9.366 & 8 & .312 & -.072 & -.134 \\
\hline Motivation & 10.375 & 8 & .240 & -.029 & .009 \\
\hline Interact between students & 14.642 & 8 & $.066 * *$ & .126 & .102 \\
\hline Interact instructor-student & 16.245 & 8 & $.039 *$ & -.046 & -.083 \\
\hline Discipline & 5.524 & 8 & .700 & .083 & .037 \\
\hline Cheat & 9.090 & 8 & .169 & -.079 & -.107 \\
\hline Self-directed & 8.178 & 8 & .416 & .082 & .065 \\
\hline Independence & 6.719 & 8 & .567 & -.037 & -.060 \\
\hline Schedule flexibility & 9.988 & 8 & .266 & .195 & .174 \\
\hline Time investment & 3.198 & 8 & .921 & .015 & -.013 \\
\hline Cost investment & 4.345 & 6 & .630 & -.241 & -.222 \\
\hline Preference opposite? & 3.179 & 4 & .528 & .006 & -.018 \\
\hline Happiness with environment & 6.051 & 8 & .641 & -.012 & -.028 \\
\hline Appropriateness & 5.763 & 4 & .218 & -.127 & -.069 \\
\hline
\end{tabular}

$* \mathrm{p} \leq .05 \quad * * \mathrm{p} \leq .10$ 
Table 3. FTF Instructors by Level Taught

\begin{tabular}{|c|c|c|c|c|c|}
\hline Metric & $\begin{array}{l}\text { Pearson Chi- } \\
\text { Square Value }\end{array}$ & Df & $\begin{array}{l}\text { Asymptotic } \\
\text { Significance } \\
\text { (2-sided) }\end{array}$ & $\begin{array}{c}\text { Pearson's } \\
\text { R }\end{array}$ & $\begin{array}{l}\text { Spearman } \\
\text { Correlation }\end{array}$ \\
\hline Difficulty & 1.56 & 4 & .816 & .059 & .057 \\
\hline Motivation & 6.029 & 3 & .110 & -.258 & -.210 \\
\hline Interact between students & 1.994 & 3 & .574 & -.149 & -.181 \\
\hline Interact instructor-student & 1.786 & 3 & .618 & -.027 & -.013 \\
\hline Discipline & 6.486 & 3 & $.090 * *$ & .115 & .161 \\
\hline Cheat & 8.906 & 3 & $.031 *$ & -.221 & -.158 \\
\hline Self-directed & 1.454 & 4 & .835 & -.039 & -.088 \\
\hline Independence & 3.545 & 3 & .315 & -.134 & -.154 \\
\hline Schedule flexibility & 6.039 & 4 & .196 & -.204 & -.176 \\
\hline Time investment & .568 & 3 & .904 & -.029 & -.011 \\
\hline Cost investment & 1.962 & 3 & .580 & .052 & .036 \\
\hline Preference opposite? & .957 & 2 & .620 & -.150 & -.153 \\
\hline Happiness with environment & .510 & 2 & .775 & -.029 & -.006 \\
\hline Appropriateness & 4.706 & 2 & $.095 * *$ & -.132 & -.170 \\
\hline
\end{tabular}

$* \mathrm{p} \leq .05 \quad * * \mathrm{p} \leq .10$ 
Table 4. OL Instructors by \# of Years Taught at University

\begin{tabular}{|c|c|c|c|c|c|}
\hline Metric & $\begin{array}{l}\text { Pearson Chi- } \\
\text { Square Value }\end{array}$ & Df & $\begin{array}{l}\text { Asymptotic } \\
\text { Significance } \\
\text { (2-sided) }\end{array}$ & $\begin{array}{c}\text { Pearson's } \\
\text { R }\end{array}$ & $\begin{array}{l}\text { Spearman } \\
\text { Correlation }\end{array}$ \\
\hline Difficulty & 94.645 & 88 & .295 & -.015 & -.013 \\
\hline Motivation & 77.258 & 88 & .787 & .128 & .092 \\
\hline Interact between students & 94.369 & 88 & .302 & .094 & .112 \\
\hline Interact instructor-student & 83.048 & 88 & .629 & .108 & .122 \\
\hline Discipline & 76.540 & 88 & .803 & .045 & .046 \\
\hline Cheat & 45.706 & 66 & .973 & .040 & -.004 \\
\hline Self-directed & 89.104 & 88 & .447 & .099 & .113 \\
\hline Independence & 96.742 & 88 & .246 & -.115 & -.093 \\
\hline Schedule flexibility & 94.235 & 88 & .305 & -.012 & .016 \\
\hline Time investment & 91.195 & 88 & .387 & .053 & .021 \\
\hline Cost investment & 57.960 & 66 & .749 & .080 & .078 \\
\hline Preference opposite? & 44.520 & 44 & .450 & -.020 & -.030 \\
\hline Happiness with environment & 82.949 & 88 & .632 & .026 & .028 \\
\hline Appropriateness & 47.334 & 44 & .338 & -.142 & -.165 \\
\hline
\end{tabular}

$* \mathrm{p} \leq .05 \quad * * \mathrm{p} \leq .10$ 
Table 5. FTF Instructors by \# of Years Taught at University

\begin{tabular}{|c|c|c|c|c|c|}
\hline Metric & $\begin{array}{l}\text { Pearson Chi- } \\
\text { Square Value }\end{array}$ & Df & $\begin{array}{l}\text { Asymptotic } \\
\text { Significance } \\
\text { (2-sided) }\end{array}$ & $\begin{array}{c}\text { Pearson's } \\
\text { R }\end{array}$ & $\begin{array}{l}\text { Spearman } \\
\text { Correlation }\end{array}$ \\
\hline Difficulty & 79.249 & 84 & .626 & -.142 & -.131 \\
\hline Motivation & 62.874 & 60 & .375 & .276 & .257 \\
\hline Interact between students & 74.000 & 60 & .106 & .054 & -.003 \\
\hline Interact instructor-student & 50.741 & 40 & .119 & .052 & .042 \\
\hline Discipline & 50.920 & 60 & .792 & -.113 & -.031 \\
\hline Cheat & 63.20 & 60 & .364 & -.006 & .054 \\
\hline Self-directed & 82.337 & 84 & .531 & .106 & .088 \\
\hline Independence & 56.207 & 60 & .615 & .222 & .188 \\
\hline Schedule flexibility & 90.169 & 80 & .205 & -.117 & -.132 \\
\hline Time investment & 64.641 & 63 & .419 & -.075 & .003 \\
\hline Cost investment & 71.275 & 60 & .151 & -.279 & -.222 \\
\hline Preference opposite? & 51.831 & 42 & .142 & .045 & .069 \\
\hline Happiness with environment & 49.109 & 42 & .210 & .131 & .119 \\
\hline Appropriateness & 41.600 & 42 & .488 & -.191 & -.162 \\
\hline
\end{tabular}

$* \mathrm{p} \leq .05 \quad * * \mathrm{p} \leq .10$ 
Table 6. OL Instructors by \# of Years Taught at Overall

\begin{tabular}{|c|c|c|c|c|c|}
\hline Metric & $\begin{array}{l}\text { Pearson Chi- } \\
\text { Square Value }\end{array}$ & Df & $\begin{array}{l}\text { Asymptotic } \\
\text { Significance } \\
\text { (2-sided) }\end{array}$ & $\begin{array}{c}\text { Pearson's } \\
\text { R }\end{array}$ & $\begin{array}{l}\text { Spearman } \\
\text { Correlation }\end{array}$ \\
\hline Difficulty & 109.359 & 96 & .166 & .077 & .100 \\
\hline Motivation & 86.726 & 96 & .740 & .209 & .209 \\
\hline Interact between students & 103.108 & 96 & .292 & -.079 & -.090 \\
\hline Interact instructor-student & 120.033 & 96 & $.049 *$ & .084 & .098 \\
\hline Discipline & 110.822 & 96 & .143 & .045 & -.012 \\
\hline Cheat & 95.127 & 72 & $.035 *$ & .069 & .031 \\
\hline Self-directed & 107.560 & 96 & .197 & .205 & .214 \\
\hline Independence & 122.349 & 96 & $.036 *$ & .110 & .161 \\
\hline Schedule flexibility & 87.458 & 96 & .721 & .121 & .144 \\
\hline Time investment & 97.784 & 96 & .430 & .095 & .017 \\
\hline Cost investment & 81.990 & 72 & .197 & .114 & .130 \\
\hline Preference opposite? & 41.948 & 48 & .718 & -.023 & .020 \\
\hline Happiness with environment & 103.357 & 96 & .286 & .061 & .086 \\
\hline Appropriateness & 49.330 & 48 & .420 & -.116 & -.121 \\
\hline
\end{tabular}

$* \mathrm{p} \leq .05 \quad * * \mathrm{p} \leq .10$ 
Table 7. FTF Instructors by \# of Years Taught at Overall

\begin{tabular}{|c|c|c|c|c|c|}
\hline Metric & $\begin{array}{l}\text { Pearson Chi- } \\
\text { Square Value }\end{array}$ & Df & $\begin{array}{l}\text { Asymptotic } \\
\text { Significance } \\
\text { (2-sided) }\end{array}$ & $\begin{array}{c}\text { Pearson's } \\
\text { R }\end{array}$ & $\begin{array}{l}\text { Spearman } \\
\text { Correlation }\end{array}$ \\
\hline Difficulty & 97.711 & 88 & .225 & -.110 & -.075 \\
\hline Motivation & 50.444 & 66 & .922 & .262 & .233 \\
\hline Interact between students & 60.588 & 66 & .665 & .013 & -.053 \\
\hline Interact instructor-student & 53.552 & 44 & .153 & .046 & .000 \\
\hline Discipline & 62.315 & 66 & .606 & -.154 & -.171 \\
\hline Cheat & 69.793 & 66 & .351 & .014 & -.008 \\
\hline Self-directed & 80.926 & 88 & .691 & .081 & .054 \\
\hline Independence & 61.229 & 66 & .643 & .210 & .195 \\
\hline Schedule flexibility & 91.725 & 88 & .372 & -.128 & -.125 \\
\hline Time investment & 74.429 & 66 & .223 & -.177 & -.171 \\
\hline Cost investment & 62.729 & 66 & .591 & -.221 & -.145 \\
\hline Preference opposite? & 39.081 & 44 & .682 & .050 & .051 \\
\hline Happiness with environment & 49.635 & 44 & .259 & .122 & .083 \\
\hline Appropriateness & 48.197 & 44 & .307 & -.156 & -.127 \\
\hline
\end{tabular}

$* \mathrm{p} \leq .05 \quad * * \mathrm{p} \leq .10$ 
Table 8: OL versus Took an OL Course

\begin{tabular}{|c|c|c|c|c|c|}
\hline Metric & $\begin{array}{l}\text { Pearson Chi- } \\
\text { Square Value }\end{array}$ & Df & $\begin{array}{c}\text { Asymptotic Significance } \\
\text { (2-sided) }\end{array}$ & $\begin{array}{c}\text { Pearson's } \\
\text { R }\end{array}$ & $\begin{array}{l}\text { Spearman } \\
\text { Correlation }\end{array}$ \\
\hline Difficulty & 4.026 & 4 & .403 & -.184 & -.213 \\
\hline Motivation & 2.453 & 4 & .653 & -.019 & -.003 \\
\hline $\begin{array}{c}\text { Interact between } \\
\text { students }\end{array}$ & 3.676 & 4 & .452 & -.074 & -.088 \\
\hline $\begin{array}{c}\text { Interact instructor- } \\
\text { student }\end{array}$ & 3.778 & 4 & .437 & -.255 & -.270 \\
\hline Discipline & 12.316 & 4 & $.015 *$ & -.379 & -.430 \\
\hline Cheat & .195 & 3 & .978 & -.041 & -.025 \\
\hline Self-directed & 8.266 & 4 & $.082 * *$ & .001 & -.012 \\
\hline Independence & 6.051 & 4 & .195 & -.051 & -.111 \\
\hline Schedule flexibility & 1.756 & 4 & .781 & .041 & .015 \\
\hline Time investment & 10.982 & 4 & $.027 *$ & -.180 & -.275 \\
\hline Cost investment & 2.372 & 3 & .499 & -.138 & -.136 \\
\hline Preference opposite? & 5.726 & 2 & $.057 * *$ & .072 & .094 \\
\hline $\begin{array}{l}\text { Happiness with } \\
\text { environment }\end{array}$ & 6.179 & 4 & .186 & .021 & .000 \\
\hline Appropriateness & .643 & 2 & .725 & -.119 & -.105 \\
\hline
\end{tabular}

$* \mathrm{p} \leq .05 \quad * * \mathrm{p} \leq .10$ 
Table 9. FTF versus Took an Online Course at University

\begin{tabular}{|c|c|c|c|c|c|}
\hline Metric & $\begin{array}{l}\text { Pearson Chi- } \\
\text { Square Value }\end{array}$ & Df & $\begin{array}{c}\text { Asymptotic Significance } \\
\text { (2-sided) }\end{array}$ & $\begin{array}{c}\text { Pearson's } \\
\text { R }\end{array}$ & $\begin{array}{l}\text { Spearman } \\
\text { Correlation }\end{array}$ \\
\hline Difficulty & 2.388 & 4 & .665 & -.125 & -.128 \\
\hline Motivation & 7.110 & 3 & $.068 * *$ & .181 & .163 \\
\hline $\begin{array}{c}\text { Interact between } \\
\text { students }\end{array}$ & 4.031 & 3 & .258 & .083 & .036 \\
\hline $\begin{array}{c}\text { Interact instructor- } \\
\text { student }\end{array}$ & 6.313 & 3 & $.097 * *$ & .269 & .197 \\
\hline Discipline & 1.180 & 3 & .758 & .083 & .070 \\
\hline Cheat & 4.329 & 3 & .228 & .328 & .321 \\
\hline Self-directed & 2.234 & 4 & .693 & .088 & .131 \\
\hline Independence & 2.969 & 3 & .398 & .266 & .262 \\
\hline Schedule flexibility & 2.504 & 4 & .644 & .073 & .082 \\
\hline Time investment & 5.343 & 3 & .148 & .184 & .188 \\
\hline Cost investment & 1.128 & 3 & .770 & .146 & .144 \\
\hline Preference opposite? & 7.063 & 2 & .029 & .407 & .372 \\
\hline $\begin{array}{l}\text { Happiness with } \\
\text { environment }\end{array}$ & .607 & 2 & .738 & .078 & .057 \\
\hline Appropriateness & 1.814 & 2 & .404 & .164 & .179 \\
\hline
\end{tabular}

$* \mathrm{p} \leq .05 \quad * * \mathrm{p} \leq .10$ 
Table 10: OL versus Took OL Course Elsewhere

\begin{tabular}{|c|c|c|c|c|c|}
\hline Metric & $\begin{array}{l}\text { Pearson Chi- } \\
\text { Square Value }\end{array}$ & Df & $\begin{array}{c}\text { Asymptotic } \\
\text { Significance (2-sided) }\end{array}$ & $\begin{array}{c}\text { Pearson's } \\
\text { R }\end{array}$ & $\begin{array}{l}\text { Spearman } \\
\text { Correlation }\end{array}$ \\
\hline Difficulty & 2.995 & 4 & .565 & .196 & .176 \\
\hline Motivation & 5.869 & 4 & .209 & .022 & .005 \\
\hline $\begin{array}{c}\text { Interact between } \\
\text { students }\end{array}$ & 6.118 & 4 & .190 & .199 & .221 \\
\hline $\begin{array}{c}\text { Interact instructor- } \\
\text { student }\end{array}$ & 5.344 & 4 & .254 & .098 & .129 \\
\hline Discipline & 6.459 & 4 & .167 & .303 & .271 \\
\hline Cheat & 3.791 & 3 & .285 & -.039 & -.010 \\
\hline Self-directed & .234 & 4 & .994 & -.020 & -.007 \\
\hline Independence & 3.739 & 4 & .443 & .041 & -.002 \\
\hline Schedule flexibility & 5.015 & 4 & .286 & -.328 & -.323 \\
\hline Time investment & 7.519 & 4 & .111 & .215 & .184 \\
\hline Cost investment & 2.280 & 3 & .516 & .052 & .088 \\
\hline Preference opposite? & 1.408 & 2 & .495 & -.181 & -.183 \\
\hline $\begin{array}{l}\text { Happiness with } \\
\text { environment }\end{array}$ & 2.516 & 4 & .642 & -.013 & -.023 \\
\hline Appropriateness & 1.592 & 2 & .451 & -.134 & -.166 \\
\hline
\end{tabular}


Table 11. FTF versus Took OL Course Elsewhere

\begin{tabular}{|c|c|c|c|c|c|}
\hline Metric & $\begin{array}{l}\text { Pearson Chi- } \\
\text { Square Value }\end{array}$ & Df & $\begin{array}{c}\text { Asymptotic } \\
\text { Significance (2-sided) }\end{array}$ & $\begin{array}{c}\text { Pearson's } \\
\text { R }\end{array}$ & $\begin{array}{l}\text { Spearman } \\
\text { Correlation }\end{array}$ \\
\hline Difficulty & 4.988 & 4 & .289 & .000 & .012 \\
\hline Motivation & 1.130 & 3 & .770 & -.145 & -.150 \\
\hline $\begin{array}{c}\text { Interact between } \\
\text { students }\end{array}$ & 3.280 & 3 & .350 & -.135 & -.082 \\
\hline $\begin{array}{c}\text { Interact instructor- } \\
\text { student }\end{array}$ & 2.486 & 3 & .478 & -.096 & -.042 \\
\hline Discipline & 2.587 & 3 & .460 & -.096 & -.081 \\
\hline Cheat & .980 & 3 & .806 & -.080 & -.072 \\
\hline Self-directed & .377 & 4 & .984 & -.059 & -.036 \\
\hline Independence & 1.816 & 3 & .611 & .189 & .189 \\
\hline Schedule flexibility & .310 & 4 & .989 & .007 & .014 \\
\hline Time investment & 2.623 & 3 & .453 & .192 & .187 \\
\hline Cost investment & 2.190 & 3 & .534 & .212 & .216 \\
\hline Preference opposite? & 7.112 & 2 & $.029 *$ & .355 & .288 \\
\hline $\begin{array}{l}\text { Happiness with } \\
\text { environment }\end{array}$ & 1.378 & 2 & .502 & .024 & -.014 \\
\hline Appropriateness & 3.378 & 2 & .185 & .022 & .057 \\
\hline
\end{tabular}

$* \mathrm{p} \leq .05 \quad * * \mathrm{p} \leq .10$ 
Table 12. OL Instructors by Self-reported Technology Level

\begin{tabular}{|c|c|c|c|c|c|}
\hline Metric & $\begin{array}{l}\text { Pearson Chi- } \\
\text { Square Value }\end{array}$ & Df & $\begin{array}{c}\text { Asymptotic } \\
\text { Significance (2-sided) }\end{array}$ & $\begin{array}{c}\text { Pearson's } \\
\text { R }\end{array}$ & $\begin{array}{l}\text { Spearman } \\
\text { Correlation }\end{array}$ \\
\hline Difficulty & 9.018 & 8 & .341 & .023 & .076 \\
\hline Motivation & 7.038 & 8 & .533 & .083 & .064 \\
\hline $\begin{array}{c}\text { Interact between } \\
\text { students }\end{array}$ & 6.641 & 8 & .576 & .152 & .161 \\
\hline $\begin{array}{c}\text { Interact instructor- } \\
\text { student }\end{array}$ & 8.438 & 8 & .392 & .249 & .253 \\
\hline Discipline & 10.231 & 8 & .249 & .053 & .094 \\
\hline Cheat & 8.925 & 6 & .178 & .264 & .310 \\
\hline Self-directed & 15.303 & 8 & $.054 * *$ & .342 & .387 \\
\hline Independence & 13.555 & 8 & $.094 * *$ & .296 & .348 \\
\hline Schedule flexibility & 10.310 & 8 & .244 & .204 & .273 \\
\hline Time investment & 8.167 & 8 & .417 & .101 & .185 \\
\hline Cost investment & 7.211 & 6 & .302 & -.094 & -.056 \\
\hline Preference opposite? & 8.425 & 4 & $.077 * *$ & .418 & .409 \\
\hline $\begin{array}{c}\text { Happiness with } \\
\text { environment }\end{array}$ & 13.914 & 8 & $.084 * *$ & .357 & .390 \\
\hline Appropriateness & 4.842 & 4 & .304 & -.331 & -.313 \\
\hline
\end{tabular}

$* \mathrm{p} \leq .05 \quad * * \mathrm{p} \leq .10$ 
Table 13. FTF Instructors by Self-reported Technology Level

\begin{tabular}{|c|c|c|c|c|c|}
\hline Metric & $\begin{array}{l}\text { Pearson Chi- } \\
\text { Square Value }\end{array}$ & Df & $\begin{array}{c}\text { Asymptotic } \\
\text { Significance (2-sided) }\end{array}$ & $\begin{array}{c}\text { Pearson's } \\
\text { R }\end{array}$ & $\begin{array}{l}\text { Spearman } \\
\text { Correlation }\end{array}$ \\
\hline Difficulty & 13.599 & 12 & .327 & .119 & .135 \\
\hline Motivation & 10.205 & 9 & .334 & -.246 & -.225 \\
\hline $\begin{array}{c}\text { Interact between } \\
\text { students }\end{array}$ & 6.153 & 9 & .725 & -.283 & -.237 \\
\hline $\begin{array}{c}\text { Interact instructor- } \\
\text { student }\end{array}$ & 6.217 & 9 & .718 & -.039 & -.026 \\
\hline Discipline & 12.844 & 9 & .170 & .101 & .127 \\
\hline Cheat & 5.475 & 9 & .791 &.-195 & -.186 \\
\hline Self-directed & 11.110 & 12 & .520 & -.081 & -.152 \\
\hline Independence & 18.513 & 9 & $.030 *$ & -.348 & -.337 \\
\hline Schedule flexibility & 16.045 & 12 & .189 & -.325 & -.305 \\
\hline Time investment & 14.566 & 9 & .104 & -.099 & -.076 \\
\hline Cost investment & 9.156 & 9 & .423 & .272 & .242 \\
\hline Preference opposite? & 3.351 & 6 & .764 & -.266 & -.272 \\
\hline $\begin{array}{l}\text { Happiness with } \\
\text { environment }\end{array}$ & 2.965 & 6 & .813 & -.159 & -.121 \\
\hline Appropriateness & 15.814 & 6 & $.015 *$ & -.280 & -.273 \\
\hline
\end{tabular}

$* \mathrm{p} \leq .05 \quad * * \mathrm{p} \leq .10$ 


\section{Online Perspectives - Instructor Survey}

Please note: The coding is the number in parentheses following the response and will not be viewed by

the participant.

Q1. What school do you teach in?

o School of Arts and Sciences (1)

- School of Education and Human Services (2)

o School of Business (3)

Q2. How old are you?

o Less than $26(1)$

o $26-30(2)$

o $\quad 31-35(3)$

o $\quad 36-40(4)$

o $41-45(5)$

o $46-50(6)$

o $\quad 51-55(7)$

o $\quad 56-60(8)$

o $\quad 61-65(9)$

o $\quad 66-70(10)$

o 71 or older (11)

Q3. Are you:

o Male (1)

o Female (2) 
Q4. What is your rank at the University?

o Lecturer

o Assistant Professor

o Associate Professor

o Professor

o Other

Q5. Do you teach undergraduate courses only, graduate courses only or both levels?

o Undergraduate courses only (1)

o Graduate courses only (2)

o Both undergraduate and graduate courses (3)

Q6. What is your highest education level?

o Associates

o Bachelors

o Masters

o Doctorate

o Post-Doctorate

Q7. Would you classify your technological abilities as:

o Do not use technology in the classroom or teaching at all.

o Beginner/Novice

o Advanced Beginner (eg. Use some Desire2Learn features, Microsoft Office)

o Intermediate (e.g. use many Desire2Learn features in classes, Microsoft Office, other computer applications)

o Superior (Extensive knowledge and use of various computer capabilities in the classroom) 
Q8. How many years have you taught at the University?

Q9. How many years have you taught at the university level (any school)?

Q10. Have you ever taken an online course at the University?

o Yes (1)

o No (2)

Q11. Have you ever taken an online course at a school other than the University?

o Yes (1)

o No (2)

Q12. Have you taught an online course at the University?

o Yes (1)

o No (2)

Section A: Participants with online teaching experience are directed to the following questions, white participants with no ontine teaching experience are direct to section B.

With respect to teaching online courses at the University compared to face-to-face (traditional classrooms), please rate the following responses:

Q13. How many online courses have you taught at the University? 
Q14. Have you taught an online course at a school other than the University?

o Yes (1)

o No (2)

Q15. How many online courses have you taught at a school other than the University?

Q16. Prior to teaching an online course, did you take a course to prepare you for the online environment?

o Yes (1)

o No (2)

Q17. If yes, was the course offered by the University or the book publisher or other method?

o University (1)

o Book publisher (2)

o Other (3)

Q18. I perceive online courses to be in difficulty than face-to-face courses.

o Significantly easier (1)

o Easier (2)

o The Same Difficulty (3)

o Harder (4)

o Significantly Harder (5)

Q19. I am motivated in online courses than face-to-face courses.

o Significantly less (1) 
o Less (2)

o Equally (3)

o More (4)

o Significantly more (5)

Q20. I _ t the interaction with students in the online environment compared to the face-to-face course environment.

o Significantly dislike (1)

o Dislike (2)

o Equate (3)

o Like (4)

o Significantly like (5)

Q21. I find the discipline required in taking online courses to be than in face-to-face courses.

o Significantly less (1)

o Less (2)

o The Same (3)

o $\quad$ More (4)

o Significantly more (5)

Q22. I enjoy the schedule flexibility associated with the online learning environment than the interaction in face-to-face courses.

o Significantly less (1)

o Less (2)

o The Same (3)

o More (4) 
o Significantly more (5)

Q23. I find online courses require time investment in the course than face-to-face courses.

o Significantly less (1)

o Less (2)

o The Same (3)

o More (4)

o Significantly more (5)

Q24. I find online courses total costs are than face-to-face courses.

o Significantly less (1)

o Less (2)

o The Same (3)

o $\quad$ More (4)

o Significantly More (5)

Q25. I perceive that it is for students to cheat in the online environment than in face-to-face courses.

o Significantly easier (1)

o Easier (2)

o The Same (3)

o Harder (4)

o Significantly harder (5) 
Q26. I enjoy the self-directed online learning environment than the interaction in face-to-face courses.

o Significantly less (1)

o Less (2)

o The Same (3)

o $\quad$ More (4)

o Significantly more (5)

Q27. I enjoy the independence associated with the online learning environment than the interaction in face-to-face courses.

o Significantly less (1)

o Less (2)

o The Same (3)

o $\quad$ More (4)

o Significantly more (5)

Q28. In the online environment, I feel these activities increase my students' understanding of the course material. (Check all that apply.)

o Discussion boards (1)

o In-class sessions (2) 
o Additional Reading (3)

o Homework (4)

o Videos (5)

o Instructor lectures (6)

o Instructor chat (7)

o Other students (8)

o Problem Scaffolding \& Hints (9)

o Textbook (10)

o Instructor Posted Notes (11)

o Surveys (12)

Q29. In the online environment, I feel these activities decrease my students' understanding of the course material. (Check all that apply.)

o Discussion boards (1)

o In-class sessions (2)

o Reading (3)

o Homework (4)

o Videos (5)

o Instructor lectures (6)

o Instructor chat (7)

o Other students (8)

o Problem Scaffolding \& Hints (9)

o Textbook (10)

o Instructor Posted Notes (11)

o Surveys (12) 
Q30. Would you prefer to teach the class in a traditional face-to-face environment?

o Yes (1)

o Undecided (2)

o No (3)

Q31. I am ___ with the online course environment for learning.

o Not very happy (1)

o Not happy (2)

o Okay (3)

o Happy (4)

o Very happy (5)

Q32. Given this institution, do you think online courses are appropriate?

o Yes (1)

O Undecided (2)

o No (3)

Q33. Why did you choose to teach the course online?

Section B: Participants with no online teaching experience are directed to the following questions, while participants with online teaching experience are direct to Section A. 
With respect to teaching face-to-face courses (traditional) at the University compared to teaching online, please rate the following responses:

Q34. I perceive face-to-face courses to be in difficulty than online courses.

o Significantly Easier (1)

o $\quad$ Easier (2)

o The Same Difficulty (3)

o Harder (4)

o Significantly Harder (5)

Q35. I would be ___ motivated in face-to-face courses than online courses.

o Significantly less (1)

o Less (2)

o Equally (3)

o $\quad$ More (4)

o Significantly more (5)

Q36. I perceive the interaction with other students in the face-to-face environment to be compared to the online course environment.

o Significantly worse (1)

o Worse (2)

o Equal (3)

o Better (4)

o Significantly better (5) 
Q37. I perceive the interaction with the instructor in the face-to-face environment to be compared to the online course environment.

o Significantly worse (1)

o Worse (2)

o Equal (3)

o Better (4)

o Significantly better (5)

Q38 I perceive the discipline required in taking face-to-face courses to be than in online courses.

o Significantly less (1)

o Less (2)

o The Same (3)

o $\quad$ More (4)

o Significantly more (5)

Q39. I perceive that it would be to cheat in the face-to-face environment than in online courses.

o Significantly easier (1)

o Easier (2)

o The Same (3)

o Harder (4)

o Significantly harder (5)

Q40 I believe that I would enjoy the self-directed face-to-face learning environment than the interaction in online courses.

o Significantly less (1) 
o Less (2)

o The Same (3)

o More (4)

o Significantly more (5)

Q41. I believe that I would enjoy the independence associated with the face-to-face environment than the interaction in online courses.

o Significantly less (1)

o Less (2)

o The Same (3)

o $\quad$ More (4)

o Significantly more (5)

Q42. I believe that I would enjoy the schedule flexibility associated with the face-to-face learning environment ___ than in online courses.

o Significantly less (1)

o Less (2)

o The Same (3)

o $\quad$ More (4)

o Significantly more (5) 
Q43. I believe that face-to-face courses require time investment in the course than online courses.

o Significantly less (1)

o Less (2)

o The Same (3)

o More (4)

o Significantly more (5)

Q44. I believe face-to-face courses total costs are than online courses.

o Significantly less (1)

o Less (2)

o The Same (3)

o More (4)

o Significantly more (5)

Q45. In the face-to-face environment, I feel these activities increase my students' understanding of the course material. (Check all that apply.)

o Discussion boards (1)

o In-class sessions (2)

o Additional Reading (3)

o Homework (4)

o Videos (5)

o Instructor lectures (6)

0 Instructor chat (7)

o Other students (8)

o Problem Scaffolding \& Hints (9)

o Textbook (10) 
o Instructor Posted Notes (11)

o Surveys (12)

Q46 In the face-to-face environment, I feel these activities decrease the students' understanding of the course material. (Check all that apply.)

o Discussion boards (1)

o In-class sessions (2)

o Additional Reading (3)

o Homework (4)

o Videos (5)

o Instructor lectures (6)

o Instructor chat (7)

o Other students (8)

o Problem Scaffolding \& Hints (9)

o Textbook (10)

o Instructor Posted Notes (11)

o Surveys (12)

Q47 Would you prefer to teach the class in an online environment?

o Yes (1)

o Undecided (2)

o No (3)

Q48. If not, why not? 
Q49. I am with the face-to-face environment for learning.

o Not very happy (1)

o Not happy (2)

o Okay (3)

o Happy (4)

o Very happy (5)

Q50. Given this institution, do you think online courses are appropriate?

o Yes (1)

o Undecided (2)

o No (3) 